"This document is the Accepted Manuscript version of a Published Work that appeared in final form in ACS Applied Materials and Interfaces, copyright (C) American Chemical Society after peer review and technical editing by the publisher. To access the final edited and published work see: https://doi.org/10.1021/acsami.6b02529" 


\title{
Polymer Enhanced Stability of Inorganic Perovskite Nanocrystals and Their Application in Color Conversion LEDs
}

Michaela Meyns, ${ }^{\dagger}$ Mariano Perálvarez, ${ }^{\dagger}$ Amelie Heuer-Jungemann, ${ }^{\perp}$ Wim Hertog, ${ }^{\dagger}$ Maria Ibáñez,

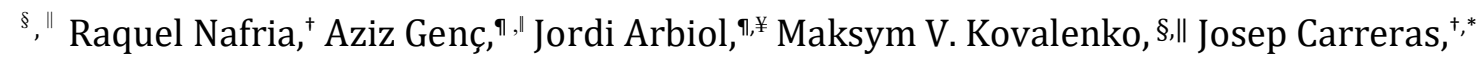
Andreu Cabot, ${ }^{+, \ldots, *}$ Antonios G. Kanaras ${ }^{\perp, *}$

† Catalonia Institute for Energy Research - IREC, Sant Adrià de Besòs, Barcelona, o8930, Spain.

${ }^{\perp}$ Physics and Astronomy, Faculty of Physical Sciences and Engineering, University of Southampton, Highfield, Southampton, $\mathrm{SO}_{17}$ 1BJ, U.K.

' Metallurgy and Materials Engineering Department, Faculty of Engineering, Bartin University, 7410o, Bartin, Turkey

I Catalan Institute of Nanoscience and Nanotechnology (ICN2), CSIC and The Barcelona Institute of Science and Technology (BIST), Campus UAB, Bellaterra, o8193 Barcelona, Spain.

${ }^{¥}$ ICREA, Pg. Lluís Companys 23, o8o1o Barcelona, Spain.

$\S$ Institute of Inorganic Chemistry, Department of Chemistry and Applied Biosciences, ETH Zürich, CH-8o93, Switzerland

I| Empa-Swiss Federal Laboratories for Materials Science and Technology, Dübendorf, CH-86oo, Switzerland

\begin{abstract}
Cesium lead halide ( $\mathrm{CsPbX}, \mathrm{X}=\mathrm{Cl}, \mathrm{Br}, \mathrm{I})$ nanocrystals ( $\mathrm{NCs}$ ) offer exceptional optical properties for several potential applications but their implementation is hindered by a low chemical and structural stability and limited processability. In the present work, we developed a new method to efficiently coat $\mathrm{CsPbX}_{3} \mathrm{NCs}$, which resulted in their increased chemical and optical stability as well as processability. The method is based on the incorporation of poly(maleic anhydride-alt-1-octadecene) (PMA) into the synthesis of the perovskite NCs. The presence of PMA in the ligand shell stabilizes the NCs by tightening the ligand binding, limiting in this way the NC surface interaction with the surrounding media. . We further show that these NCs can be embedded in self-standing silicone/glass plates as down-conversion filters for the fabrication of monochromatic green and white light emitting diodes (LEDs) with narrow bandwidths and appealing color characteristics.
\end{abstract}

Keywords: Inorganic perovskite nanocrystals, $\mathrm{CsPbBr}_{3}$, LED, color conversion, poly(maleic anhydride-alt-1octadecene)

*Corresponding authors

Josep Carreras: jcarreras@irec.cat

Andreu Cabot: acabot@irec.cat

Antonios Kanaras: a.kanaras@soton.ac.uk 


\section{INTRODUCTION}

Inorganic lead halide perovskite NCs with high luminescence quantum yields (up to $>90 \%$ ), extremely narrow emission bandwidths ( $<50 \mathrm{~nm}$ ), broad emission spectra tunability (from 410 to $700 \mathrm{~nm}$ ) and short radiative lifetimes ( $<30 \mathrm{~ns}$ ) have very recently emerged as a new class of material with outstanding potential for optoelectronic applications. ${ }^{1-3}$ Compared to classical $\mathrm{Cd}$-based chalcogenide quantum dots (QDs), $\mathrm{CsPbX}_{3}(\mathrm{X}=\mathrm{Cl}, \mathrm{Br}, \mathrm{I})$ offer a very broad and facile adjustable composition versatility together with ample options for shape control, which allow tuning of their emission wavelength throughout the whole visible spectrum. ${ }^{4-9}$ Among other applications, such highly luminescent and spectrally tunable NCs are ideally suited to produce monochromatic and white LEDs (WLEDs). ${ }^{10}$ In this regard, $\mathrm{CsPbBr}_{3} \mathrm{NCs}$ have been utilized as the emissive layer in electroluminescent monochromatic QD-LEDs showing exceptionally narrow emission bandwidths and thus high color quality in devices. ${ }^{11,12}$

However, while $\mathrm{CsPbX}_{3}(\mathrm{X}=\mathrm{Br}, \mathrm{l})$ perovskite $\mathrm{NCs}$ can be stored in solution, maintaining their luminescence for several months, they suffer from humidity, light and temperature driven degradation in operation conditions and from a low colloidal stability with a related limited processability. ${ }^{13}$ In part, this is associated with a highly dynamic ligand binding to the NC surface, ${ }^{14}$ which additionally hinders the NCs' post-synthetic purification and further functionalization. When perovskite NCs with highly ionic components and high surface energies come into contact with a polar surface or solvent, they rapidly degrade to their components. Any efforts to exchange the oleic acid coating of the as prepared NCs with ligands of interest providing additional functionality, rendering them water soluble or just protecting them from degradation, inevitably results in the NC degradation. This chemical instability is the bottleneck that limits all technological applications where the NC processing into a composite or layer is required and where NCs are exposed to an external source of energy for long times. Very recently, a step towards better stabilization was achieved by crosslinking of the long-chain ligands oleic acid and oleylamine while attached to the NC surface by highly energetic radiation. ${ }^{15}$ Additionally, the interdigitation of octadecene molecules was reported to increase stability and emission properties of organohalide perovskite NCs. ${ }^{16}$ This raised the hope that an enforced ligand sphere is able to enhance the stability of inorganic perovskite NC.

The integration of $\mathrm{CsPbX}_{3} \mathrm{NCs}$ in color conversion instead of electroluminescent LEDs is considered a more convenient and, in terms of material stability and processability, less demanding strategy to fabricate NC LEDs. With this approach, typical problems occurring in electroluminescent LEDs such as charging and thermal stress are reduced as no current flows through the layer. Still the processability of color conversion LEDs is not straightforward. The conventional procedure to fabricate a color conversion LED using NCs as optical phosphors involves the blending of the NCs with a thermo-curable silicone resin and deposition on top of the pump LED. In this procedure, the presence of hydrophobic organic ligand and solvents hamper the polymerization of the encapsulating resin and can reduce the mechanical stability of the composite. This is especially problematic in lead halide perovskites requiring an excess of organic ligands in solution to prevent their aggregation, ${ }^{14}$ which would reduce their $Q Y$ and introduce scattering thus decreasing light emision. ${ }^{17}$ To overcome the hydrophobicity limitation and improve dispersion, ligand exchange processes, the growth of oxide shells or the encapsulation of QDs in polymers, silica or glass matrices have been used in other types of semiconductors (e.g. ${ }^{18-20}$ ). However, the limited stability and processability of perovskite NCs have so far prevented applying similar strategies.

In order to facilitate the processing and applicability of perovskite NCs, we developed a method to produce cesium lead halide NCs with improved chemical stability and compatibility for LED encapsulation by in situ addition of poly(maleic anhydride-alt-1-octadecene) (PMA). PMA has been shown to be an ideal ligand for the encapsulation of oleic acid or oleylamine-coated inorganic NCs such as magnetic, semiconductor and 
gold nanoparticles. ${ }^{23,24}$ This ligand interdigitates in the carbon chain shell that surrounds the nanoparticle offering an enhanced stability. In the case of a stable NC core, the PMA units can be further polymerized utilizing a diamine, which allows the sealing of the NC core and its transfer to polar solvents. In our experiments, PMA was introduced during the synthesis of the perovskite NCs, before the injection of the Cs-precursor, to avoid any potential disruption of the perovskite NC after its formation. We further show the application of green- and orange-red-emitting $\mathrm{CsPbX}_{3}$ perovskite NCs embedded in self-standing silicone-based plates as down-conversion filters in monochromatic and WLEDs. The growth of the NCs in the presence of a protecting polymer facilitates their processing by preventing aggregation and minimizing interference with the silicone curing process while better protecting the NC surface both by preventing interaction with the media and by obstructing the ligand dynamic exchange.

\section{Materials and methods}

Materials: $\mathrm{PbBr}_{2}$ (99.999\% trace metals basis), 1-octadecene (90\%), oleic acid (90\%), $\mathrm{PMA}\left(\mathrm{M}_{\mathrm{n}}\right.$ : 30,000$50,000)$, fluorescein, rhodamine $6 \mathrm{G}$ and $\mathrm{Cs}_{2} \mathrm{CO}_{3}$ (99.9\% trace metals basis) were purchased from Sigma Aldrich, $\mathrm{Pbl}_{2}(99.999+\%-\mathrm{Pb})$ from Strem and oleylamine (primary amine content $\left.80-90 \%\right)$ from Acros. Polastosil-2000 curing and catalyst were obtained from Silikony Polskie. Round window glass with $27 \mathrm{~mm}$ (green LEDs) and $28 \mathrm{~mm}$ in diameter (WLEDs) was obtained from Edmund optics and Thermo Scientific.

Methods: $\mathrm{CsPbX}_{3} \mathrm{NCs}$ synthesis: $\mathrm{CsPbX}_{3} \mathrm{NCs}$ were prepared following a modified procedure from that reported by Protesescu et al. ${ }^{2}$ Lead halide salts $(0.376 \mathrm{mmol}$, green NCs: $138 \mathrm{mg} \mathrm{PbBr}$, orange-red NCs: $69 \mathrm{mg} \mathrm{PbBr}_{2}$ and $87 \mathrm{mg} \mathrm{Pbl}_{2}$ ) were mixed with 1-octadecene $(10 \mathrm{~mL})$ and heated under vacuum (60$100 \mathrm{mTorr}$ ) to $90^{\circ} \mathrm{C}$ for 2 hours. After switching to Ar atmosphere and heating to $120^{\circ} \mathrm{C}$, oleic acid (1.0 mL) and oleylamine $(1.0 \mathrm{~mL}$ ) were injected. When a clear solution was obtained, poly (maleic anhydride-alt-1octadecene) (430 mg) was added under Ar flow. Vacuum was applied and the temperature was kept constant at $100^{\circ} \mathrm{C}$ for 10 minutes. The solution was stirred vigorously (1100 rpm) while the temperature was increased to $175^{\circ} \mathrm{C}$. Once this temperature was reached, Cs-oleate solution $(0.8 \mathrm{~mL})$ with a temperature of $130^{\circ} \mathrm{C}$ was injected swiftly using a syringe pre-warmed in an oven. This way, the Cs-oleate solution remained clear, whereas partial precipitation of the precursor was observed at lower temperatures. After $15 \mathrm{~s}$ the reaction was quenched by cooling with a water bath.

Purification:

After $15 \mathrm{~s}$ the reaction was quenched by cooling with a water bath. The reaction solution was centrifuged at a speed of $6300 \mathrm{rcf}$ for 15 minutes. After decantation of the supernatant and of liquid residues eluting from the resting precipitate while leaving the centrifuge tube rest atop, the NCs were transferred to the glove box and stored in Ar atmosphere for further use (these are our samples after 1 precipitation). In our experience this was not necessary for the samples with PMA but to maintain comparable conditions we stored all samples in the glove box (for an impression of the stability of particles without and with polymer see figure S7). If further cleaning was applied, around $65 \mathrm{mg}$ of sample were dispersed in $1 \mathrm{~mL}$ toluene and precipitated by the addition of $0.3 \mathrm{~mL}$ acetonitrile and centrifugation at $10000 \mathrm{rpm}$ for three minutes. This was repeated two to three times as indicated.

Filter plate fabrication: Green filters were prepared by drop casting $6.6 \mathrm{mg}$ of NCs in $200 \mu \mathrm{L}$ of toluene onto window glass with $27 \mathrm{~mm}$ diameter and letting toluene evaporate slowly in a semi-closed petri-dish under ambient conditions. To fabricate WLEDs, dried particles of each color (Balanced LED as in Figure 5a: green 
$7.5 \mathrm{mg}$, orange $5.1 \mathrm{mg}$; LED with higher red/orange content as in Figure 6: green $7.6 \mathrm{mg}$, orange $7.8 \mathrm{mg}$ ) were dispersed with 2 drops of hexane, mixed separately with $414 \pm 5 \mathrm{mg}$ of liquid silicone Polastosil-2000 and sonicated for 5 minutes. Afterwards, $31 \mathrm{mg}$ of curing catalyst were added and the mixtures were stirred thoroughly. After ten minutes, the mixtures were deposited on spherical optical glass with $28 \mathrm{~mm}$ diameter and left to cure for 60 to $75 \mathrm{~min}$. When the silicone was nearly cured another glass slide was carefully put on top of one layer for separation before the second layer containing particles of the other color was stacked on top with the silicone layer facing the stack. The stack was left to cure fully for at least ten minutes more.

Characterization: Structural and chemical characterization: X-ray diffraction analyses were carried out on Bruker AXS D8 ADVANCE X-ray diffractometer with $\mathrm{Cu}-\mathrm{K} \alpha$ radiation $(\lambda=0.15406 \AA$ ) $)$. Reference patterns were calculated from crystallographic data of the cubic and orthorhombic phases reported in ref. ${ }^{21}$ by CaRIne software. Size and shape of the initial NCs were examined by transmission electron microscopy (TEM) using a ZEISS LIBRA 120, operating at $120 \mathrm{kV}$. High resolution TEM (HRTEM) and scanning TEM (STEM) images were recorded using an FEI Tecnai F20 TEM microscope, equipped with a high angle annular dark field (HAADF) detector, operated at $200 \mathrm{kV}$. For TEM analysis the NCs were dispersed in toluene and drop casted onto a carbon-coated copper grid. Field-emission scanning electron microscopy (SEM) was carried out on an Auriga Zeiss at $5.0 \mathrm{kV}$. Quantitative elemental analysis was performed by means of energy dispersive X-ray spectroscopy (EDX) within the SEM. Metal contents were determined with Perkin Elmer inductively coupled plasma (ICP) instruments using mass spectrometry (Nexlon 350D) for Cs and atomic emission spectroscopy for $\mathrm{Pb}$ (Optima 8300). Samples synthesized in reactions with an upscaling factor of two for all substances were purified as reported earlier. ${ }^{14}$ Of each purified and dried sample (2 per batch) around $30 \mathrm{mg}$ were attacked by adding $5 \mathrm{~mL}$ of $\mathrm{HNO}_{3}$ and $3 \mathrm{~mL}$ of $\mathrm{H}_{2} \mathrm{O}_{2}$ and heated until $210{ }^{\circ} \mathrm{C}$ in a microwave. Finally, the samples were diluted to $50 \mathrm{~mL}$ with ultrapure water and measured.

For optical characterization the samples were diluted in hexane and analyzed using a quartz cuvette with $1 \mathrm{~cm}$ path length on a Cary 300 Bio UV-vis Spectrophotometer and a Cary Eclipse Fluorescence Spectrophotometer. Quantum yields were determined according to literature procedures using Rhodamine $6 \mathrm{G}$ and Fluorescein as standards. ${ }^{22}$ Each QY was determined from measurements in three NC batches produced under the same conditions and with at least 2 measurements per batch.

Nuclear Magnetic Resonance (NMR) measurements were recorded on a Bruker Avance III HD Spectrometer operating at a $1 \mathrm{H}$ frequency of $500.26 \mathrm{MHz}$ and equipped with a BBFO-Z probe. For NMR measurements batches scaled up with a factor 2 were produced and purified by two cycles of precipitation and redispersion with acetonitrile/toluene before drying and redispersing them in deuterated toluene. The sample temperature was set to $298.2 \mathrm{~K}$. One dimensional (1D) 1H and 2D NOESY (Nuclear Overhauser Effect Spectroscopy) spectra were acquired using standard pulse sequences from the Bruker library. For the quantitative $1 \mathrm{D} 1 \mathrm{H}$ measurements, $64 \mathrm{k}$ data points were sampled with the spectral width set to $20 \mathrm{ppm}$ and a relaxation delay of $30 \mathrm{~s}$. NOESY mixing time was set to $300 \mathrm{~ms}$ and 4096 data points in the direct dimension for 512 data points in the indirect dimension were typically sampled, with the spectral width set to $10 \mathrm{ppm}$. Diffusion measurements (2D DOSY) were performed using a double stimulated echo sequence for convection compensation and with monopolar gradient pulses. (ref. J. Magn. Reson. 2009, 198, 121-131) Smoothed rectangle gradient pulse shapes were used throughout. The gradient strength was varied linearly from 2 to $95 \%$ of the probe's maximum value in 64 increments, with the gradient pulse duration and diffusion delay optimized to ensure a final attenuation of the signal in the final increment of less than $10 \%$ relative to the first increment. For $2 \mathrm{D}$ processing, the spectra were zero filled until a 
4096-2048 real data matrix. Before Fourier transformation, the 2D spectra were multiplied with a squared cosine bell function in both dimensions, the 1D spectra were multiplied with an exponential window function. The diffusion coefficients were obtained by fitting the appropriate Stejskal- Tanner equation to the signal intensity decay. (ref. Concepts Magn. Reson., Part A 2012, 40A, 39-65) Diffusion measurements (2D DOSY) were performed using a double stimulated echo sequence for convection compensation and with monopolar gradient pulses; dstegp2s. ${ }^{1}$ (Connell, M. A.; Bowyer, P. J.; Bone, P. A.; Davis, A. L.; Swanson, A. G.; Nilsson, M.; Morris, G. A., Improving the accuracy of pulsed field gradient NMR diffusion experiments: Correction for gradient non-uniformity. J. Magn. Reson. 2009, 198 (1), 121-131) Smoothed rectangle gradient pulse shapes were used throughout. The gradient strength was varied linearly from $2-95 \%$ of the probe's maximum value (calibrated at $50.2 \mathrm{G} / \mathrm{cm}$ ) in 64 steps, with the gradient pulse duration and diffusion delay optimized to ensure a final attenuation of the signal in the final increment of less than $10 \%$ relative to the first increment. The diffusion coefficients were obtained by fitting the Stejskal-Tanner (ST) equation to the signal intensity decay. For the pulse sequence at hand, the appropriate ST equation is: 2 (Sinnaeve, D., The Stejskal-Tanner equation generalized for any gradient shape-an overview of most pulse sequences measuring free diffusion. Concepts Magn. Reson. Part A 2012, 40A (2), 39-65.)

$$
I=I_{0} e^{-(\gamma \delta g \xi)^{2} D(\Delta-0.6 \delta)}
$$

with the gyromagnetic ratio of the observed ${ }^{1} \mathrm{H}$ nucleus $\gamma$, the gradient pulse length $\delta$, the gradient strength $g$, the diffusion time $\Delta$ and the diffusion coefficient $D$. The shape factor $\xi$ depends on the gradient shape but this is usually already corrected for in the gradient strength value by the Bruker software, TOPSPIN. The ST equation for other pulse programs is only slightly different and the impact on the diffusion coefficient is very small in the case of long diffusion times ( $\Delta>250 \mathrm{~ms}$ ). In a real DOSY experiment, values of $\Delta$ and $\delta$ are set while varying $g$ to derive the decay curve.

LED testing: The measurements were carried out under ambient conditions. For green LEDs a Thorlabs' Mounted High-Power LED M365L2 UV pump with $0.55 \mathrm{~W}$ (3.42 V, I= $156.4 \mathrm{~mA}$ ) was applied to illuminate the filter. The detection of the resulting luminescence was performed by means of an ISP 500 integrating sphere and a SPECTRO 320 PMT (Photomultiplier tube) based spectrometer, both from Instrument Systems. WLEDs were evaluated in an ISP 2000 integrating sphere coupled to a CAS 120 CCD-based spectrometer, both from Instrument Systems. The blue pump $(450 \mathrm{~nm})$ was run at $5.48 \mathrm{~V}$ and $58.8 \mathrm{~mA}$, which implied a total power consumption of $0.31 \mathrm{~W}$. The total irradiated filter area had a diameter of $25 \mathrm{~mm}$. It is worth noticing that the switch from $365 \mathrm{~nm}$ to $450 \mathrm{~nm}$ as pump wavelength responded to the necessity of complementing the emission from the filter (by covering the short wavelength range) in the research of white light. In addition, it should be noted that both spectrophotometric systems are equivalent. The change from one system to the other is related to the fact that, for mechanical reasons, the blue light from the $450 \mathrm{~nm}$ pump was more easily coupled to the ISP 2000 sphere.

\section{RESULTS AND DISCUSSION}

Figure 1 shows representative TEM micrographs of the cubic green-emitting $\mathrm{CsPbBr}_{3}$ and cubic/partially squared orange-red-emitting $\left.\mathrm{CsPbBr}_{1.6}\right|_{1.4} \mathrm{NCs}$ obtained in the presence of PMA ( $\mathrm{CsPbX}_{3}-\mathrm{PMA}$ ) following the procedure above described. Fig. $1 \mathrm{C}$ and $1 \mathrm{D}$ respectively shows an atomic resolution HAADF STEM micrograph of one $\mathrm{NC}$ and its corresponding power spectrum, confirming the cubic crystal structure of the NC. The prepared $\mathrm{CsPbX}_{3}-\mathrm{PMA}$ NCs thus exhibited the same crystal structure and a very similar emission 
maximum as those prepared in the same conditions by the unmodified synthesis protocol. $\mathrm{CsPbBr}_{3}-\mathrm{PMA}$ and $\mathrm{CsPbBr}_{1.6} \mathrm{l}_{1.4}$-PMA NCs had average edge lengths of $9 \pm 1 \mathrm{~nm}$ and $11 \pm 2 \mathrm{~nm}$ outside the quantum confinement regime, ${ }^{2}$ first absorption maxima at 504 and $561 \mathrm{~nm}$ and corresponding emission wavelengths of 513 and $586 \mathrm{~nm}$.

SEM-EDX and ICP analyses revealed the $\mathrm{CsPbX}_{3}$-PMA NCs to have slightly non-stoichiometric compositions, with an excess of lead and halide providing atomic ratios $\mathrm{Cs}: \mathrm{Pb}: \mathrm{Br}=1: 1.2: 4$ for green-emitting $\mathrm{NCs}$ and $\mathrm{Cs}: \mathrm{Pb}: \mathrm{Br}: \mathrm{I}=1: 1.1: 2: 1.4$ for orange-red NCs (see supporting information for details). This off-stoichiometry may arise from the fact that the reaction is carried out under an excess of the two elements which may entangle with the polymer. An excess of halide may further be attributed to its role in the surface capping of the NCs, where it constitutes the negative counterpart of oleylammonium ions that stabilize the NCs in solution. ${ }^{14}$ The NC quantum yield (QY) increased slightly but reproducibly with the polymer addition for both the green-emitting $\mathrm{CsPbBr}_{3} \mathrm{NCs}$, from $49 \pm 4 \%$ to $53 \pm 4 \%$, and the red-emitting $\left.\mathrm{CsPbBr}_{1.6}\right|_{1.4} \mathrm{NCs}$, from $84 \pm 3 \%$ to $88 \pm 1 \%$. We believe the reason behind this slight increase is a better passivation of the trap states by the more stable ligand shell. ${ }^{14}$
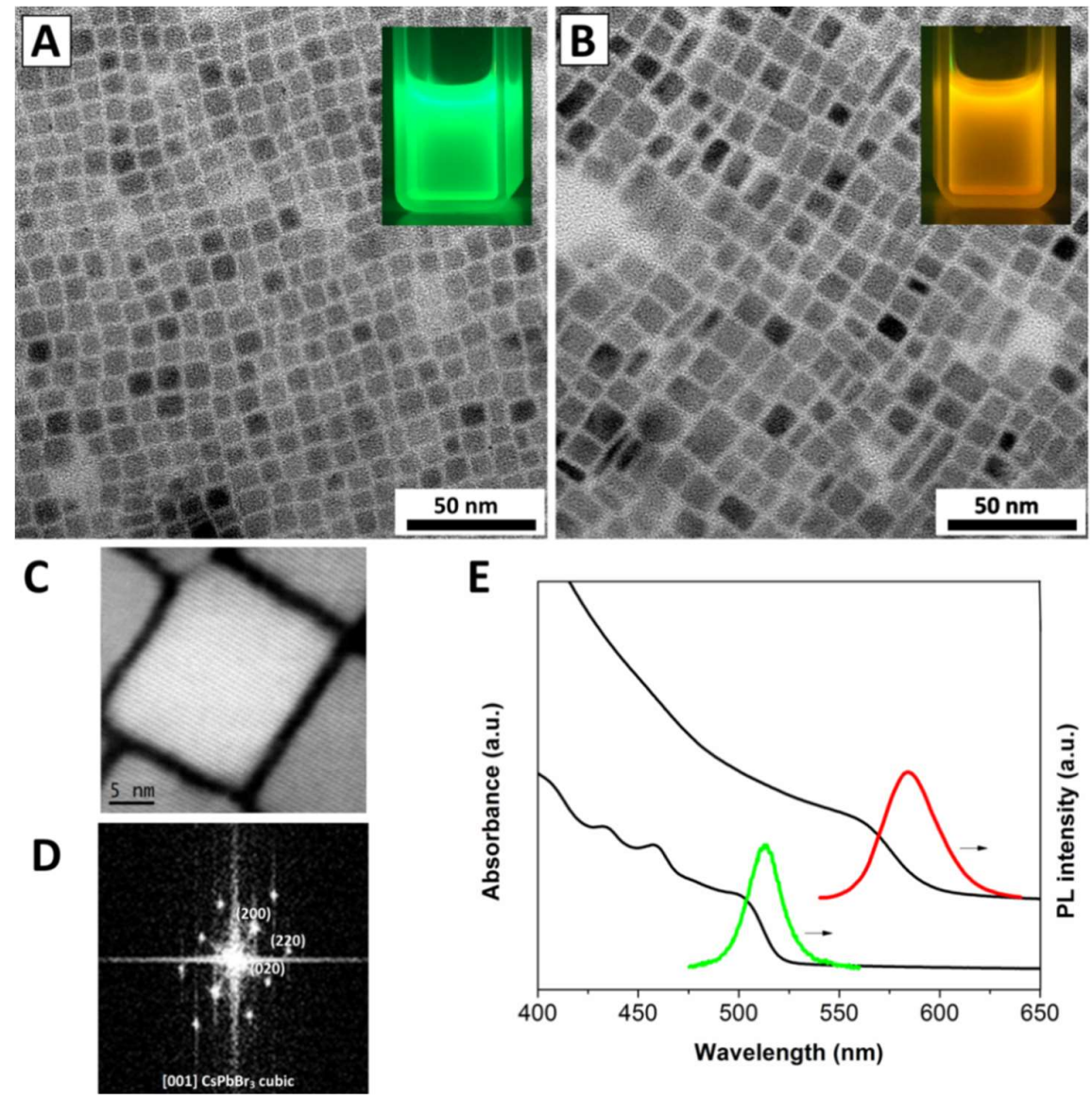

Figure 1. TEM micrographs of $A$ ) green $\mathrm{CsPbBr}_{3}$ and $\mathrm{B}$ ) orange $\mathrm{CsPbBr}{ }_{1.6} \mathrm{l}_{1.4} \mathrm{NCs}$. C) Atomic resolution HAADF micrograph of one $\mathrm{CsPbBr}_{3} \mathrm{NC}$ and $\mathrm{D}$ ) its power spectrum confirming a cubic crystal structure. E) Absorbance and emission spectra of the NCs in toluene solution.

To investigate this point further, we studied the composition of the ligand shell by means of ${ }^{1} \mathrm{H}$ solution NMR and IR spectroscopy (Figures S5 and S6). The surface study by means of ${ }^{1} \mathrm{H}$ solution NMR showed the 
presence of oleylammonium bromide or oleylammonium oleate as surface ligands, in both the samples produced with and without the presence of PMA. The overlapping of the resonances between these ligands and the PMA, prevented us to directly identify the presence of PMA in the ${ }^{1} \mathrm{H}$ NMR spectrum. Additionally, the similarity of the spectrum of PMA to the ligands and the fact that the anhydride ring seems to open during the reaction providing more $-\mathrm{COOH}$ and $-\mathrm{COO}^{-}$groups that are positioned close to oleic acid/oleate signals, did neither allow to unambiguously identify the presence of PMA on the samples by IR spectroscopy. However, Diffusion Ordered NMR Spectroscopy (DOSY) clearly revealed a change of the dynamic behaviour of those ligands in the presence of PMA. Lower diffusion coefficients and thus a solvodynamic radius closer to the NC size, were systematically obtained for all samples containing PMA (Table 1). This indicates that when polymer is added to the solution, ligands are indeed more tightly bound to the NCs and it indirectly proves the presence of PMA on the NPs surface. While original ligands are still present, we presume that PMA is entangled on the ligand shell and acts as a contingency wall reducing the kinetics of the dynamic stabilization. On the other hand, NMR analysis showed that adding PMA after the synthesis was not so effective. Adding PMA to a pure $\mathrm{CsPbBr}_{3}$ sample after the synthesis combined with sonication $10 \mathrm{~min}$ and purification with acetonitrile resulted in larger diffusion coefficients when compared with purified samples synthesized in the presence of PMA (table 1). Besides, the addition of PMA under such circumstances presented a photoluminescence decay already visible by eye. This underlines the importance of adding PMA during the synthesis and not after.

Table 1. Diffusion coefficient and solvodynamic cube edges of $\mathrm{CsPbBr}_{3} \mathrm{NCs}$ produced with or without the presence of PMA.

\begin{tabular}{|l|c|c|c|}
\hline \multicolumn{1}{|c|}{ Sample } & $\begin{array}{c}\text { Diffusion Coefficient } \\
\left(\mu \mathrm{m}^{2} \mathrm{~s}^{-1}\right)\end{array}$ & $\begin{array}{c}\text { TEM size } \\
(\mathrm{nm})\end{array}$ & $\begin{array}{c}\text { Solvodynamic size } \\
(\mathrm{nm})\end{array}$ \\
\hline $\mathrm{CsPbBr}_{3}$ & 89 & $9.4 \pm 1.5$ & 6.9 \\
\hline $\mathrm{CsPbBr}_{3}-\mathrm{PMA}$ & 64 & $9.7 \pm 1.7$ & 9.5 \\
\hline $\mathrm{CsPbBr}_{3}(\mathrm{PMA})^{*}$ & 90 & $11 \pm 2$ & 6.8 \\
\hline
\end{tabular}

* PMA added after synthesis

A main challenge to fabricate color conversion QD-WLEDs is to increase durability by preventing the NC degradation with a prolonged LED operation period. QD-LED degradation can be the result of a loss of QD luminescence when integrated into the device, due to photo-, thermal- or chemical degradation and to aggregation during processing due to incompatibility between the surface organic ligands and the polymer matrix. We tested the effect of the PMA on the stability of $\mathrm{CsPbX}_{3} \mathrm{NCs}$ when used as down converters of UV light. For this application, a toluene solution of $\mathrm{CsPbX}_{3}-\mathrm{PMA} \mathrm{NCs}$ was spread on $27 \mathrm{~mm}$ diameter glass substrates followed by slow evaporation of toluene in a semi-closed container to obtain a homogeneous layer.

These plates were placed on top of a commercial $365 \mathrm{~nm}$ UV-emitting LED delivering a radiometric power of $100 \mathrm{~mW}_{\text {opt. }}$. Under these conditions the $\mathrm{CsPbBr}_{3}-\mathrm{PMA} \mathrm{NCs}$ emitted at $528 \mathrm{~nm}$ with a full-width at half maximum of $17 \mathrm{~nm}$. The green-emitting LED without PMA had a color saturation of 0.944 , a luminous efficacy of radiation of $255 \mathrm{~lm} / \mathrm{W}_{\text {opt. }}$ and a luminous efficacy of $2.5 \mathrm{~lm} / \mathrm{W}_{\text {elec. }}\left(1 \mathrm{~mW}\right.$ opt.. $\left./ W_{\text {electr. }}\right)$. Devices incorporating $\mathrm{CsPbBr}_{3}-\mathrm{PMA} \mathrm{NCs}$ provided a color saturation increased to 0.988 and a much higher luminous efficacy of radiation up to $562 \mathrm{~lm} / \mathrm{W}_{\text {opt }}$, associated to its spectral proximity to the maximum of the photopic curve $\left(683 \mathrm{~lm} / \mathrm{W}_{\mathrm{opt}}\right.$, at $555 \mathrm{~nm}$ ) and the partial UV light absorption by the polymer. The luminous efficacy was, in contrast, slightly lower, $1.8 \mathrm{~lm} / \mathrm{W}_{\text {elec. }}$. 
Figure 2 shows the temporal evolution of the spectra obtained from two filters produced from the same amount of $\mathrm{CsPbBr}_{3} \mathrm{NCs}$ and thoroughly purified $\mathrm{CsPbBr}_{3}-\mathrm{PMA} \mathrm{NCs}$ under continuous illumination by a $365 \mathrm{~nm}$ pump in ambient conditions. This pump was operated at $58.8 \mathrm{~mA}$ forward current and provided an optical power of $100 \mathrm{~mW}_{\text {opt. }}$ at sample level. In both cases, the luminescence decreased with time and shifted to longer wavelengths. The stability of the emission signal was significantly higher for the $\mathrm{CsPbBr}_{3}-$ PMA NCs, with $60 \%$ of peak area remaining after twelve operation hours, compared to $\mathrm{CsPbBr}_{3} \mathrm{NCs}$ with no PMA protection, decreasing down to $21 \%$.
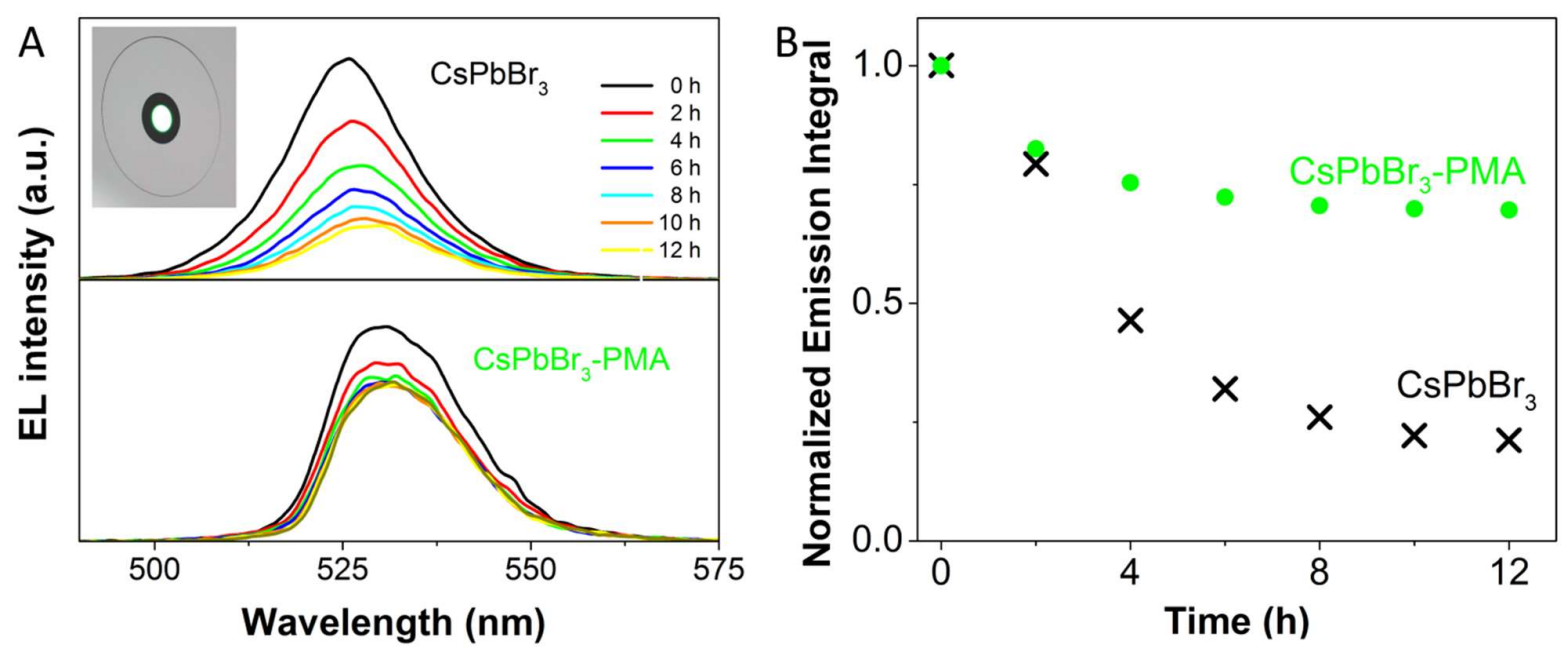

Figure 2. A) Temporal evolution of the emission spectra measured with filters of $\mathrm{CsPbBr}_{3}$ and $\mathrm{CsPbBr}_{3}-\mathrm{PMA}$ on a UV- LED with $100 \mathrm{~mW}$ at $365 \mathrm{~nm}$ with $58.8 \mathrm{~mA}$ forward current. The inset shows a green filter in the sample position of the integrating sphere. B) Normalized integrals of the emission peaks between 460 and $600 \mathrm{~nm}$ showing a three times lower decrease of the emission signal with PMA within twelve hours of constant irradiation.

X-ray diffraction (XRD) analysis (Figure 3) suggested that the NCs grow and partially rearrange crystallographically during long-term optical excitation. This growth and rearrangement was evidenced after $22 \mathrm{~h}$ operation for $\mathrm{CsPBBr}_{3} \mathrm{NCs}$ and after 3 days for $\mathrm{CsPbBr}_{3}-\mathrm{PMA} \mathrm{NCs}$ and was indicated by a narrowing of the XRD peaks following an extended illumination period. This growth and a rearrangement of the atoms through a phase transition would also explain the observed emission spectral red-shift. TEM micrographs in figure $\mathrm{S} 8$ show the grown crystals. The polymer effectively slowed down these processes, but it did not completely prevent them. 


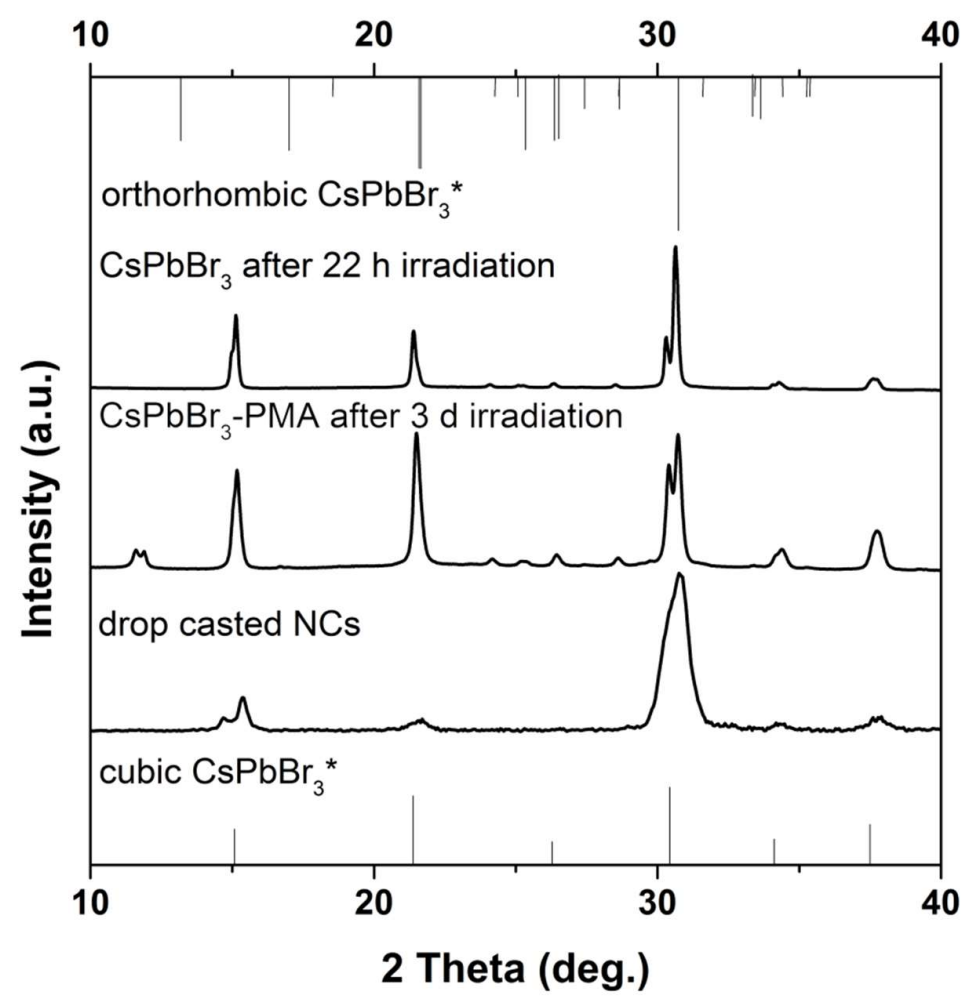

Figure 3. XRD patterns before and after long-term irradiation of $22 \mathrm{~h}\left(\mathrm{CsPbBr}_{3}\right)$ and 3 days ( $\left.\mathrm{CsPbBr}_{3}-\mathrm{PMA}\right)$. The initial NC deposited on a Si wafer by drop casting exhibit a cubic crystal structure with characteristically broad reflexes, no significant differences were observed for NC films with and without PMA. After the stability tests smaller peak widths indicate crystal growth and new peaks can be assigned to orthorhombic $\mathrm{CsPbBr}_{3}$ ( ${ }^{*}$ references were calculated based on data from ref. ${ }^{21}$, see experimental) or monoclinic [Li et al.].

To investigate the performance of $\mathrm{CsPbX}_{3} \mathrm{NCs}$ in color conversion WLEDs, we combined green-emitting $\mathrm{CsPbBr}_{3}-\mathrm{PMA}$ and red-emitting $\left.\mathrm{CsPbBr}_{1.6}\right|_{1.4}$-PMA NCs with emission peaks at 520 and $620 \mathrm{~nm}$ on independent free-standing plates. Owing to the sensitivity of perovskite NCs, a room temperature encapsulation and a remote-type architecture where a color conversion plate is placed on top of the LED at a distance was found to be more suitable for implementing perovskite-based color conversion LEDs. ${ }^{26} \mathrm{NCS}$ were embedded into a silicone matrix (Polastosil-2000) which was mixed with a catalyst at room temperature, spread on an optical glass substrate and left to cure. Heat-induced curing processes, widely applied in two-component silicone, proved inapplicable due to destruction of the perovskite NCs. To provide further protection from humidity and facilitate handling, the filter was covered by another glass layer when the silicone was mostly but not fully cured. The applied silicone is not only an easy to handle as well as cost effective matrix but also through adhesion forces towards the glass leads to a homogeneous distribution of the layer without any bubbles. Epoxy curing used for sealing perovskite solar cells was not compatible with the NCs, as the NCs degraded upon contact with the resin, most likely due to unfavorable reactions with hydroxyl groups. Sandwiching the layers between glasses further opens the possibility of totally sealing the NCs and prevents any release of toxic $\mathrm{Pb}$ compounds. Apart from these design considerations, we discarded the blending of the two types of NCs in the same layer due to fast composition equilibration in solution that is not prevented by the ligand keeping but ion permeable PMA (see Supporting information, Figure S7). Instead we sandwiched the NCs in silicone curing between two 
glass plates or stacks of two layers between three glass slides. Each silicone layer between two glasses was measured to cause a loss of optical emission of about $6 \%$, part of this amount of light could be gained with modified LED design that redirects backscattered light in combination with more specific curing strategies reducing the difference in refractive indexes.
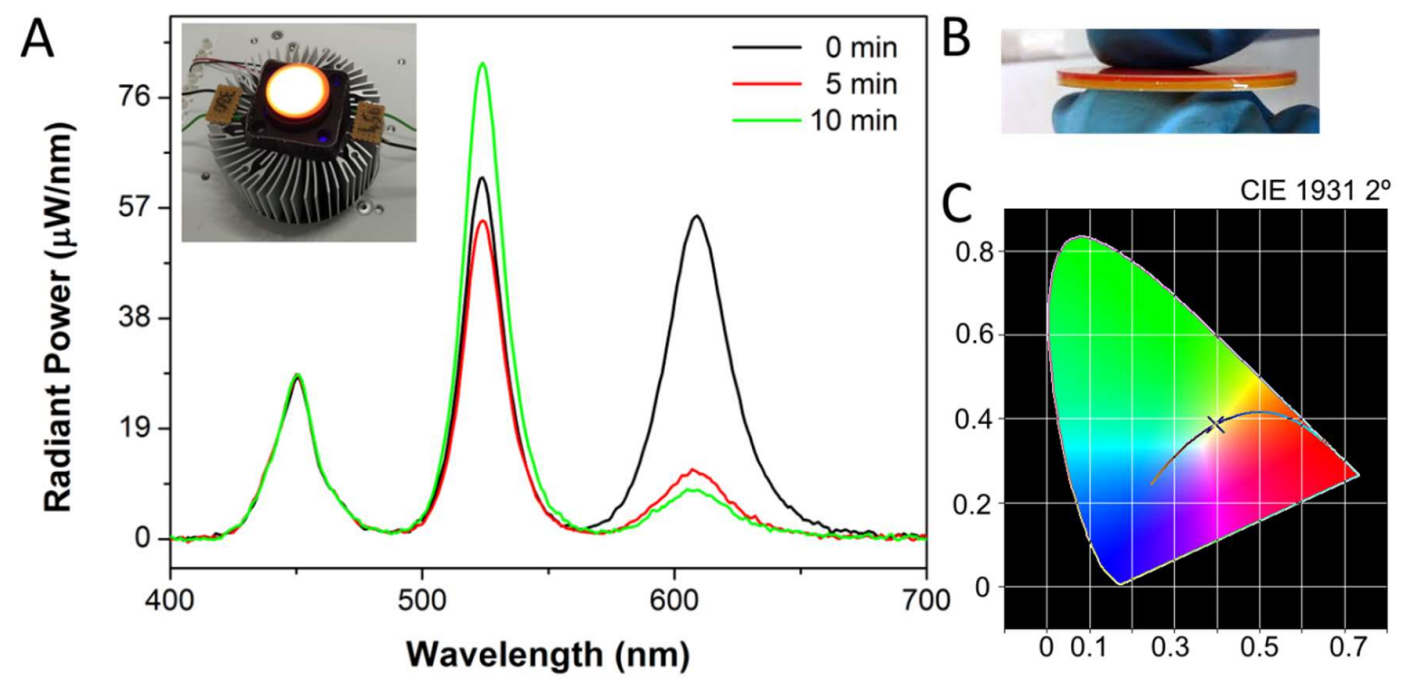

Figure 4. a) Electroluminescence spectra of a $\mathrm{CsPbX}_{3}-\mathrm{PMA}$ WLED with an initial of CRI 72.4 and CCT of $3665 \mathrm{~K}$. The inset shows a photograph of the device in the measurement set-up. B) Photograph of the stacked color conversion plates. C) CIE chromaticity diagram.

Color conversion plates containing the $\mathrm{CsPbX}_{3}$-PMA NCs were mounted over a blue-emitting $446 \mathrm{~nm}$ pump. The maximum current, $58.8 \mathrm{~mA}$ providing $100 \mathrm{~mW}_{\text {opt., }}$ was applied to test the device stability. Figure 4 shows the electroluminescence spectra obtained in ambient conditions of a WLED having the red-emitting filter stacked on top of the green-emitting one with one glass slide in between. The initial luminous efficacy of radiation (LER) of the WLED was $353 \mathrm{~lm} / \mathrm{W}_{\text {Opt }}$, which is within the range for optimum white light generation. ${ }^{10}$ However, the luminous efficacy in terms of conversion of electricity into light (LE) of $4.5 \mathrm{Im} / \mathrm{W}_{\text {elec.. }}$ leaves room for improvement. Within 5 minutes the color temperature shifted from warm/neutral white light with $3665 \mathrm{~K}$ to $9300 \mathrm{~K}$ and the CRI decreased from 72.4 to 66.9 as a consequence of the degradation of the red component. Even with the improvement accomplished by the presence of the protecting PMA, in ambient conditions and with a relatively high forward current of $60 \mathrm{~mA}$, the unstable red component limited the device durability. In iodide-based perovskite NCs photo activated atomic rearrangement is facilitated by the higher energetic gain of transition between the highly emissive cubic and less active orthorhombic phase of crystals containing iodine. ${ }^{2,25,27}$ The reason for this lies in the intrinsic properties of the anions. The lower electronegativity of iodine and its larger size makes it less stable in the cubic $\mathrm{CsPbX}_{3}$ structure, and thus the iodide-based perovskite decomposes faster than the bromide based one.(ref) In thin films recent studies show that doping with chloride ions improves the stability of the crystal phase.(ref) So far, solution based doping of the iodide containing NCs has proved evasive but future advances in this direction may help to amend the instability of the red component. During irradiation the emission of the green filter increased as the red component decreased, indicating a substantial reabsorption of the emitted green light by the red component that may be solved by advanced filter design. 
Figure 5 displays the EL spectra of a $\mathrm{CSPbX}_{3}-\mathrm{PMA}$ WLED with higher red content under different driving currents ranging from 5 to $60 \mathrm{~mA}$ taken over a period of time of 6 minutes. A non-linear increase of the red component was observed, caused by simultaneous degradation that counteracts the trend of increasing emission with higher currents. This again points out the vulnerability of the red component in comparison to the green one but on the other hand confirms the reliability of the green layer.

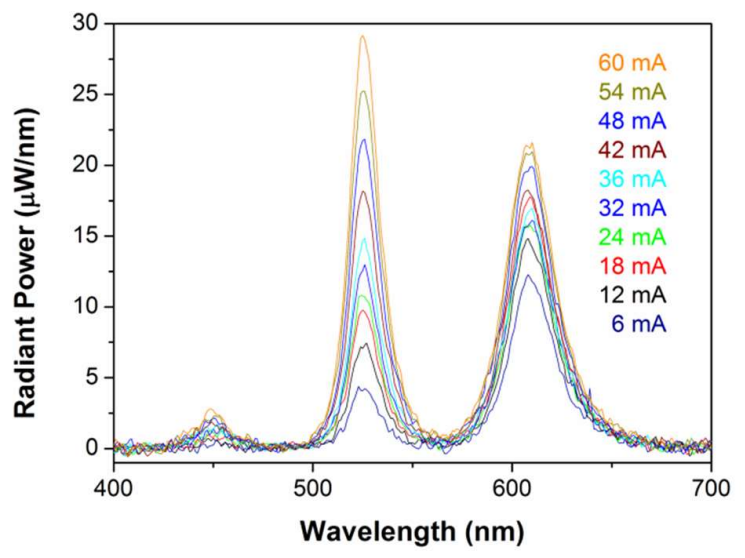

Figure 5. Electroluminescence spectra of a $\mathrm{CsPbX}_{3}-\mathrm{PMA}$ WLED with increasing applied current.

\section{CONCLUSIONS}

$\mathrm{CsPbX}_{3} \mathrm{NCs}$ were synthesized in the presence of PMA. The addition of the polymer led to an increase of QY and optical stability of $\mathrm{CsPbX}_{3} \mathrm{NCs}$ under illumination in ambient conditions which is associated to a tighter bonding of the ligands in the presence of PMA as observed by ${ }^{1} \mathrm{H}$ NMR spectroscopy. Owing to the choice of emission wavelengths and the small emission bandwidths of these NCs, color conversion LEDs with high luminous efficacies of radiation and thus bright colors were achieved. The initial values of warm/neutral white light obtained are promising, although the bottleneck of an all-CsPbX 3 color converter WLED is the orange or red component obtained with a mixed bromide/iodide composition, which even in the presence of PMA shows a limited lifetime. While further advances in NC surface passivation in combination with component encapsulation strategies and advanced LED designs are necessary, our approach suggests that growing the NCs with a compatible polymer in situ during the reaction can facilitate their further processing and may be the key for further stabilization and encapsulation procedures.

\section{Acknowledgments}

This work was supported by the European Regional Development Funds, the Framework 7 program under project UNION (FP7-NMP-2012-310250) and HI-LED (FP7-ICT-2013-11-619912) as well as the Spanish MINECO Projects BOOSTER (ENE2013-46624-C4-3-R) and AMALIE (TEC2012-38901-C02-01). M.M. thanks the Spanish MINECO for financial support through the Juan de la Cierva-formación program. A.G. and J.A. acknowledge funding from Generalitat de Catalunya 2014 SGR 1638 and the Spanish MINECO MAT201451480-ERC (e-ATOM) and Severo Ochoa Excellence Program. We would like to thank Pablo Guardia for fruitful discussions. 


\section{REFERENCES}

(1) Lin, C. C.; Meijerink, A.; Liu, R. Critical Red Components for Next-Generation White LEDs. J. Phys. Chem. Lett. 2016, 7, 495-503.

(2) Protesescu, L.; Yakunin, S.; Bodnarchuk, M. I.; Krieg, F.; Caputo, R.; Hendon, C. H.; Yang, R. X.; Walsh, A.; Kovalenko, M. V. Nanocrystals of Cesium Lead Halide Perovskites ( $\mathrm{CsPbX}, \mathrm{X}=\mathrm{Cl}, \mathrm{Br}$, and I): Novel Optoelectronic Materials Showing Bright Emission with Wide Color Gamut. Nano Lett. 2015, $15,3692-3696$.

(3) Yakunin, S.; Protesescu, L.; Krieg, F.; Bodnarchuk, M. I.; Nedelcu, G.; Humer, M.; Luca, G. De; Fiebig, M.; Heiss, W.; Kovalenko, M. V. Low-Thresold Amplified Spontaneous Emission and Lasing from Colloidal Nanocrystals of Caesium Lead Halide Perovskites. Nat. Commun. 2015, 6, 1-8.

(4) Swarnkar, A.; Chulliyil, R.; Ravi, V. K.; Irfanullah, M.; Chowdhury, A.; Nag, A. Colloidal CsPbBr 3 Perovskite Nanocrystals: Luminescence beyond Traditional Quantum Dots. Angew. Chemie 2015, $127,15644-15648$.

(5) Sun, S.; Yuan, D.; Xu, Y.; Wang, A.; Deng, Z. Ligand-Mediated Synthesis of Shape- Controlled Cesium Lead Halide Perovskite Nanocrystals via Reprecipitation Process at Room Temperature. ACS Nano 2016, ASAP, doi:10.1021/acsnano.5b08193.

(6) Nedelcu, G.; Protesescu, L.; Yakunin, S.; Bodnarchuk, M. I.; Grotevent, M. J.; Kovalenko, M. V. Fast Anion-Exchange in Highly Luminescent Nanocrystals of Cesium Lead Halide Perovskites (CsPbX $3, X=$ $\mathrm{Cl}, \mathrm{Br}, \mathrm{I})$. Nano Lett. 2015, 15, 5635-5640.

(7) Zhang, D.; Eaton, S. W.; Yu, Y.; Dou, L.; Yang, P. Solution-Phase Synthesis of Cesium Lead Halide Perovskite Nanowires. J. Am. Chem. Soc. 2015, 137, 9230-9233.

(8) Akkerman, Q. A.; Innocenzo, V. D.; Accornero, S.; Scarpellini, A.; Petrozza, A.; Prato, M.; Manna, L. Tuning the Optical Properties of Cesium Lead Halide Perovskite. J. Am. Chem. Soc. 2015, 137, 1027610281.

(9) Bekenstein, Y.; Koscher, B. A.; Eaton, S. W.; Yang, P.; Alivisatos, A. P. Highly Luminescent Colloidal Nanoplates of Perovskite Cesium Lead Halide and Their Oriented Assemblies. J. Am. Chem. Soc.C 2015, 137, 16008-16011.

(10) Erdem, T.; Demir, H. V. Color Science of Nanocrystal Quantum Dots for Lighting and Displays. 2013, 2, 57-81.

(11) Zhang, X.; Lin, H.; Huang, H.; Reckmeier, C.; Zhang, Y.; Choy, W. C. H.; Rogach, A. L. Enhancing the Brightness of Cesium Lead Halide Perovskite Nanocrystal Based Green Light-Emitting Devices through the Interface Engineering with Perfluorinated lonomer. Nano Lett. 2016, 16, 1415-1420.

(12) Song, J.; Li, J.; Li, X.; Xu, L.; Dong, Y.; Zeng, H. Quantum Dot Light-Emitting Diodes Based on Inorganic Perovskite Cesium Lead Halides (CsPbX 3 ). Adv. Mater. 2015, 27, 7162-7167. 
(13) Kim, Y.; Yassitepe, E.; Voznyy, O.; Comin, R.; Walters, G.; Gong, X.; Kanjanaboos, P.; Nogueira, A. F.; Sargent, E. H. Efficient Luminescence from Perovskite Quantum Dot Solids. ACS Appl. Mater. Interfaces 2015, 7, 25007-25013.

(14) Roo, J. De; Ibáñez, M.; Geiregat, P.; Nedelcu, G.; Walravens, W.; Maes, J.; Martins, J. C.; Driessche, I. Van; Kovalenko, M. V; Hens, Z. Highly Dynamic Ligand Binding and Light Absorption Coefficient of Cesium Lead Bromide Perovskite Nanocrystals. ACS Nano 2016, 10, 2071-2081.

(15) Palazon, F.; Akkerman, Q. A.; Prato, M.; Manna, L. X-Ray Lithography on Perovskite Nanocrystals Films: From Patterning with Anion-Exchange Reactions to Enhanced Stability in Air and Water. ACS Nano 2016, 10, 1224-1230.

(16) González-Carrero, S.; Galian, R. E.; Pérez-Prieto, J. Organometal Halide Perovskites: Bulk LowDimension Materials and Nanoparticles. Part. Part. Syst. Charact. 2015, n/a - n/a.

(17) Song, W.; Yang, H. Efficient White-Light-Emitting Diodes Fabricated from Highly Fluorescent Copper Indium Sul Fi de Core/Shell Quantum Dots. Chem. Mater. 2012, 24, 1961-1967.

(18) Kim, H.; Kwon, B.-H.; Suh, M.; Kang, D. S.; Kim, Y.; Jeon, D. Y. Degradation Characteristics of Red Light-Emitting CulnS2/ZnS Quantum Dots as a Wavelength Converter for LEDs. Electrochem. SolidState Lett. 2011, 14, K55-K57.

(19) Ziegler, B. J.; Xu, S.; Kucur, E.; Meister, F.; Batentschuk, M.; Gindele, F.; Nann, T. Silica-Coated InP / ZnS Nanocrystals as Converter Material in White LEDs. Adv. Mater. 2008, 20, 4068-4073.

(20) Song, W.; Kim, J.; Yang, H. Silica-Embedded Quantum Dots as Downconverters of Light-Emitting Diode and Effect of Silica on Device Operational Stability. Mater. Lett. 2013, 111, 104-107.

(21) Rodová, M.; Brožek, J.; Knížek, K.; Nitsch, K. Phase Transitions in Ternary Caesium Lead Bromide. J. Therm. Anal. Calorim. 2003, 71, 667-673.

(22) Grabolle, M.; Spieles, M.; Lesnyak, V.; Gaponik, N. Determination of the Fluorescence Quantum Yield of Quantum Dots : Suitable Procedures and Achievable Uncertainties. 2009, 81, 6285-6294.

(23) Di Corato, R.; Quarta, A.; Piacenza, P.; Ragusa, A.; Figuerola, A.; Buonsanti, R.; Cingolani, R.; Manna, L.; Pellegrino, T. Water Solubilization of Hydrophobic Nanocrystals by Means of Poly(maleic Anhydride- Alt-1-Octadecene). 2008, 18, 1991-1996.

(24) Bigall, N. C.; Curcio, A.; Leal, M. P.; Falqui, A.; Palumberi, D.; Di Corato, R.; Albanesi, E.; Cingolani, R.; Pellegrino, T. Magnetic Nanocarriers with Tunable pH Dependence for Controlled Loading and Release of Cationic and Anionic Payloads. Adv. Mater. 2011, 23, 5645-5650.

(25) Seth, S.; Mondal, N.; Patra, S.; Samanta, A. Fluorescence Blinking and Photoactivation of AllInorganic Perovskite Nanocrystals CsPbBr3 and CsPbBr2I. 2016, 7, 266-271.

(26) Jang, E.; Song, W.; Lee, K.; Yang, H. Preparation of a Photo-Degradation- Resistant Quantum Dot Polymer Composite Plate for Use in the Fabrication of a High-Stability White-Light-Emitting Diode. Nanotechnology 2013, 24, 045607.

(27) Park, Y.-S.; Guo, S.; Makarov, N. S.; Klimov, V. I. Room Temperature Single-Photon Emission from Individual Perovskite Quantum Dots. ACS Nano 2015, 9, 10386-10393. 